\title{
INSPIRE: Managing metadata in a Global Digital Library for High-Energy Physics
}

\author{
Javier Martin Montull on behalf of the INSPIRE collaboration \\ CERN, 1211 Geneve 23, Switzerland \\ javier.martin.montull@cern.ch
}

\begin{abstract}
Four leading laboratories in the High-Energy Physics (HEP) field are collaborating to roll-out the next-generation scientific information portal: INSPIRE. The goal of this project is to replace the popular 40 year-old SPIRES database. INSPIRE already provides access to about 1 million records and includes services such as fulltext search, automatic keyword assignment, ingestion and automatic display of LaTeX, citation analysis, automatic author disambiguation, metadata harvesting, extraction of figures from fulltext and search in figure captions. In order to achieve high quality metadata both automatic processing and manual curation are needed. The different tools available in the system use modern web technologies to provide the curators of the maximum efficiency, while dealing with the MARC standard format. The project is under heavy development in order to provide new features including semantic analysis, crowdsourcing of metadata curation, user tagging, recommender systems, integration of OAIS standards and innovative metrics.
\end{abstract}

Keywords. Digital library, high-energy physics, metadata curation

\section{Introduction}

The High-Energy Physics (HEP) community, which comprises around 30,000 researchers worldwide [1], has been using the SPIRES database [2] as one of their main information resources over the last 40 years. CERN, DESY, Fermilab and SLAC are undertaking the ambitious project of replacing the system by a more modern one that uses state-of-the-art technologies to help researchers access documentation in innovative and flexible ways.

The system is built on top of an Open Source digital library software developed at CERN, Invenio [3], currently used in over 25 institutions worldwide.

INSPIRE combines the curated content present in the SPIRES database with the materials harvested from the main information providers in the field, with arXiv.org as the primary source. The latter currently contains more than $850 \mathrm{k}$ bibliographic records.

This paper describes the functionality currently available in the INSPIRE system [4] and the development that will be incorporated in the coming years. The main focus of this article is the treatment of metadata in the system, from the initial ingestion 
to the automatic and manual curation that takes place in order to offer high quality content to scholars in the field.

\section{INSPIRE overview}

The INSPIRE system takes advantage of the features provided by the Invenio software, first deployed at CERN in 2002.

This software is based on open standards (MARCXML, MARC21, OAI-PMH) and is capable of managing any type of bibliographic entity, such as an article, paper, thesis, book, picture, video using the Machine-Readable-Cataloging (MARC) standard [5] for the representation of bibliographic information.

This digital library software is highly modular allowing the INSPIRE collaboration to develop new functionality in an agile manner. Its strength resides in the fast and powerful search engine, which uses Google-like syntax, the flexible document type submission and approval workflow, the flexible metadata treatment and the user personalization (with Web 2.0 features like baskets, notifications, user groups and user comments).

Since the INSPIRE project began, new functionalities have constantly been introduced to Invenio's code base. For instance, it is currently possible to perform searches inside the fulltexts, displaying snippets of the matches in the search page. The INSPIRE collaboration has been negotiating with publishers in order to extend this functionality to restricted articles. In April 2010, the first agreement was signed with Springer in that direction.

INSPIRE actively participates in the ORCID initiative [6], which aims to solve the author ambiguity problem that affects many fields of research. In a big step towards achieving this goal, INSPIRE deployed its own author disambiguation algorithm in the year 2011 that makes use of data such as affiliation history, coauthor information, citation patterns and more, in order to disambiguate authors with a high success rate. In Fig. 1, an author page is shown where all the name variants for a given author are displayed, together with all the important information related to the author.

In the same research track, an interface for the authors to claim their papers, which is shown in Fig. 2, has been developed in order to increase the quality of the author pages. This interface uses metadata information to group research papers that have a high probability of having been written by the given author, allowing the user to confirm/disclaim the proposals and add new papers.

Finally, the joint effort among CERN, the APS [7] and the experimental collaborations has resulted in the definition of the authors.xml format [8] that allows collaborations to present their author lists with each author being uniquely identified.

Other important features that have been put in place include the extraction of plots from arXiv.org tarballs, which allow scholars to view all plots from a record in the detailed page as well as search inside the captions (which are extracted from the LaTeX files), automatic keyword assignment and citation analysis.

Some of the citation analysis tools include citation count graphs and recommendations based on the citation network [9]. 
Ellis, John R. (903 papers)

This is me. Verify my publication list.

\begin{tabular}{l}
\hline Name variants \\
\hline Ellis, John R. (831) \\
Ellis, John (36) \\
Ellis, J. (10) \\
Ellis, John R., (ed.) (9) \\
Ellis, John R., (Ed.) (7) \\
Ellis, J.R. (6) \\
Ellis, Jonathan R. (2) \\
Ellis, John, (ed.) (1) \\
Ellis, John.R. (1)
\end{tabular}

\section{Papers}

All papers (903)

Report (903)

Published (606)

Conference (145)

Review (101)

Lectures (46)

introductory (20)

Book (7)

Thesis (2)

\section{Affiliations}

\section{CERN (823)}

SLAC (37)

unknown affiliation (28)

Caltech (9)

King's Coll. London (8)

Cambridge U.. DAMTP (7)

LBL, Berkeley (5)

Sussex U. (3)

Cambridge U. (3)

UC. Santa Cruz (2)

Oxford U. (2)

Stanford U. (1)

HARC. Woodlands (1)

Texas A-M (1)

Fermilab (1)

\section{Frequent co-authors}

Nanopoulos, Dimitri V. (222)

Olive. Keith A. (104)

Mavromatos, N.E. (73)

Karliner, Marek (24)

Gaillard. Mary K. (23)

Frequent keywords

supersymmetry (297)

Higgs particle: mass (132)

dark matter (128)

electron positron: annihilation (126)

CP: violation (94)

Enqvist. K. (21)

Gaillard, M.K. (20)

Hagelin, J.S. (19)

Heinemeyer. S. (19)

Kowalski. H. (18)

Weiglein, G. (18)

Fig. 1. Author page with author disambiguation information

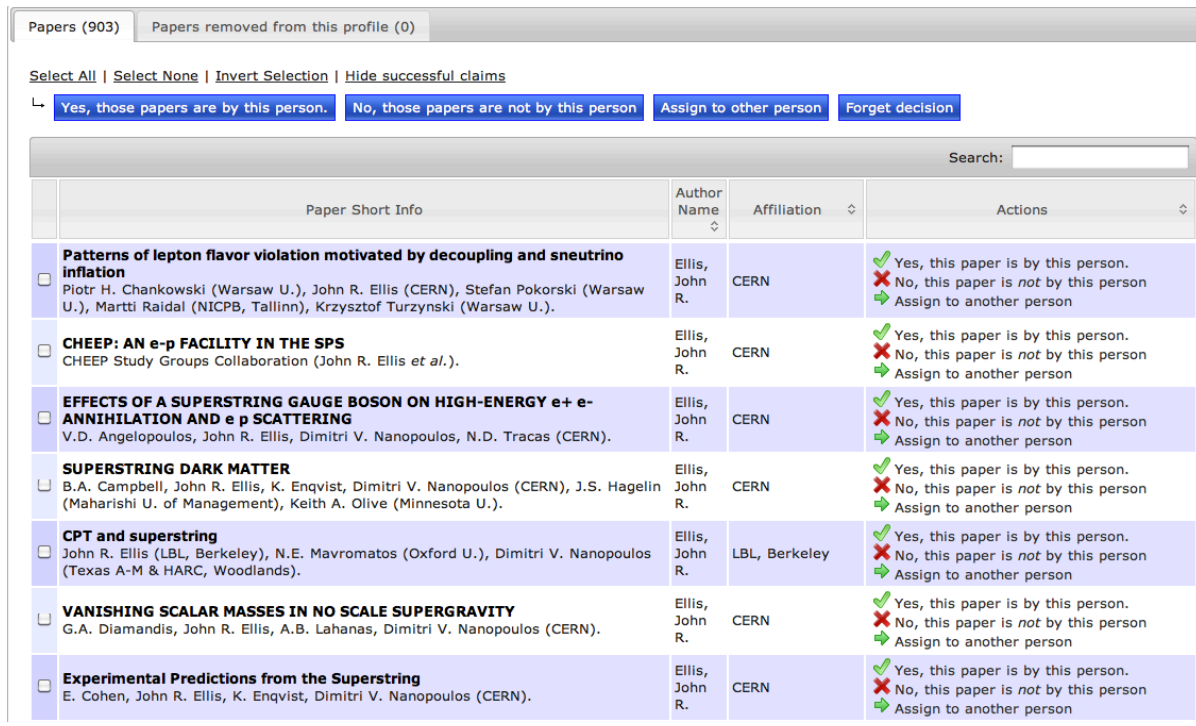

Fig. 2. Interface to claim papers for a given author 


\section{Managing metadata in INSPIRE}

The SPIRES database has remained popular over the course of decades thanks to its trusted content curated by SLAC, Fermilab and DESY. One of the main challenges that the INSPIRE system faces is providing the appropriate curation tools necessary to efficiently maintain all the metadata ingested by the system.

For INSPIRE to become independent from SPIRES, which is the final goal, it is necessary to mimic SPIRES's harvesting scheme. In addition, some filters have been designed to perform more automated classification and data-mining of incoming content.

The process starts with the harvest from external repositories and publisher feeds using common protocols (OAI-PMH) and the mapping of the harvested data to be compliant with INSPIRE's data representation, MARC21.

If the external repository provides additional files alongside the metadata, like figures or the original LaTeX source, these are extracted and indexed for user search.

To help minimize manual curation work, an automatic analysis of the fulltext files is made to extract all references and make them searchable.

Before the final insertion of the harvested record, checks are performed to avoid duplication in the database and to allow manual intervention in case of conflicts.

Once the records are part of the system, some manual curation is needed to achieve the highest possible quality in the metadata information.

All tools developed for metadata processing are Rich Internet Applications (RIAs), which allow seamless access from any given location. The technologies used include AJAX, the Python programming language and MySQL databases.

The main metadata editing tool build into the system, shown in Fig. 3, is called BibEdit and allows the editing of a single record. The record is displayed with its internal representation (MARC21) and once opened a cataloger can edit any field/subfield in a convenient way.

Every modification sent to the system creates a unique object so that any error introduced can be reverted. These revisions are shown in the interface as well, so that the cataloger can check the history of modifications, revert or merge a previous version.

In addition, useful functions such as search for records, undo/redo changes, keyboard shortcuts and preview of the record are provided.

In line with the overall goal of increasing productivity and reducing errors, new features are under development and will be available in the future iterations of INSPIRE. These include autocompletion of fields based on knowledge bases, special modes to manage citations and authors (some collaborations have thousands), and a module devoted to checking that all combinations of fields/subfields are valid.

Once the user submits the desired changes to the record, the server produces a MARCXML file, checks its compliance with the standard and sends the modification to be included in the database.

In addition to BibEdit, which only allows catalogers to correct one record at a time, a different tool named MultiEdit can apply arbitrary transformations across an entire set of records. 
MultiEdit, shown in Fig. 4, first performs a search to retrieve the set of records that the cataloger wants to correct. Once this is done, it is possible to filter the MARC tags displayed in the interface and the collection the records belong to.

After having selected the records to be modified, the interface allows the cataloger to define a set of actions that will be applied to them. The actions include adding fields/subfields, deleting them and replacing some strings among others. A preview of the modifications is then shown and all of them can be sent into the system at once.

As mentioned at the beginning of this section, the automatic harvesting will sometimes detect that the record to be added to the system is a potential duplicate. At other times a preprint is published and a new record has to be introduced with just few changes. Without an appropriate tool it is challenging for a cataloger to spot the differences between similar records and actions taken can be error prone.

Thus, INSPIRE also contains a tool named BibMerge which consists in a side-byside comparison of MARC records so that the cataloger can easily find the differences (comparison techniques such as the Levenshtein distance are used) and merge both records once the appropriate fields have been added or removed.

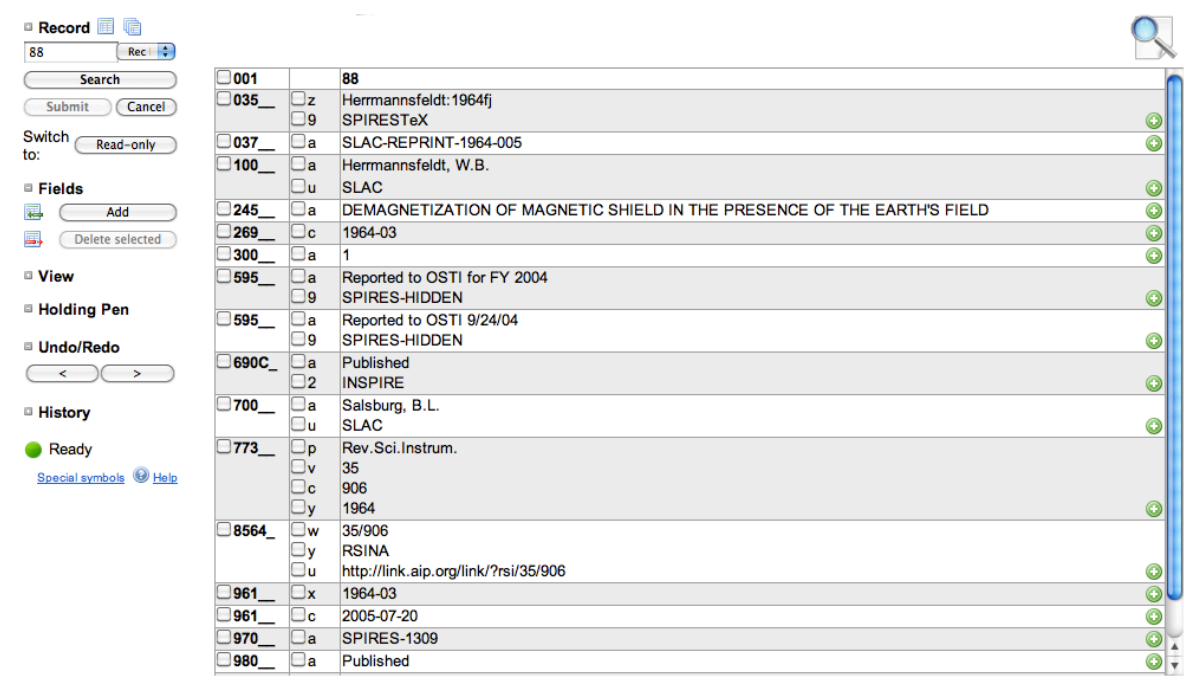

Fig. 3. BibEdit, tool for editing a metadata record 


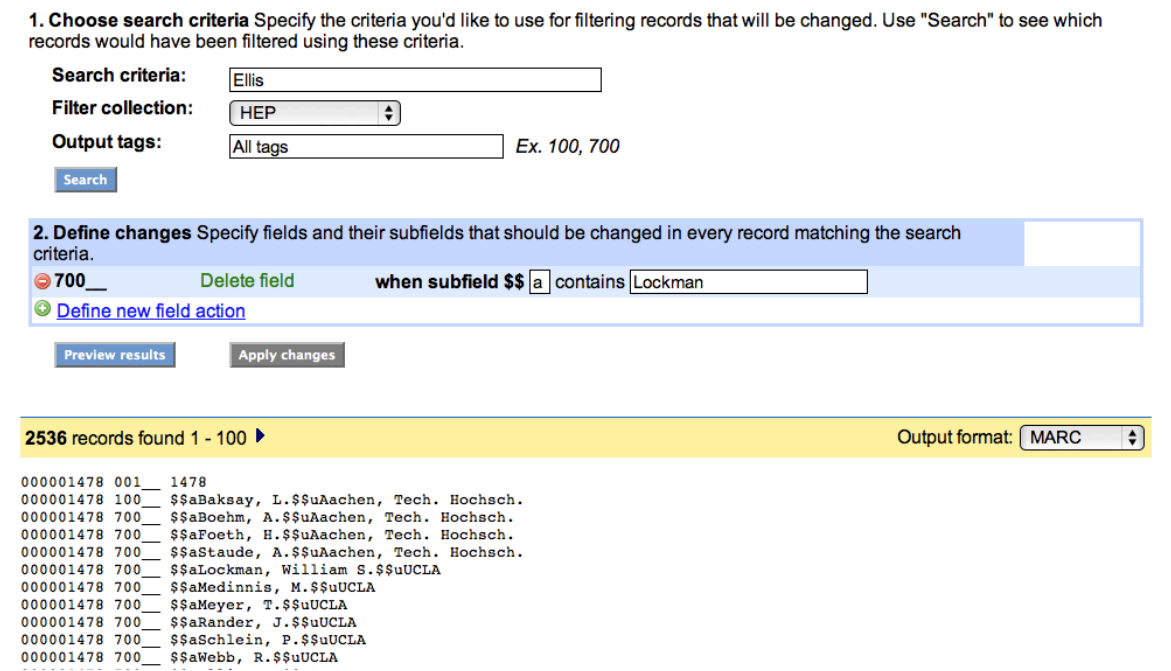

Fig. 4. MultiEdit, tool for editing multiple metadata records

\section{Conclusion}

The management of metadata in a global High-Energy Physics (HEP) digital library such as INSPIRE leads to multiple challenges, given the objective of producing highly trusted information used for research.

In order to increase the productivity of the catalogers in charge of metadata curation, all metadata harvested into the system from external sources must be preprocessed, so that as much information as possible is automatically ingested.

In cases where manual curation is required, the INSPIRE system provides a variety of tools that allow catalogers from the four participating laboratories (CERN, SLAC, DESY and Fermilab) to edit the metadata using cutting-edge technologies.

The use of these techniques allows INSPIRE to maintain high quality content and maximize the productivity of catalogers involved in the collaboration.

\section{References}

1. Travis Brooks, http://www.projecthepinspire.net/ape09.pdf (22.05.2011)

2. http://www.slac.stanford.edu/spires/

3. http://www.invenio-software.org

4. http://inspirebeta.net

5. http://www.loc.gov/standards/marcxml

6. http://www.orcid.org

7. http://www.aps.org

8. http://www.slac.stanford.edu/spires/hepnames/authors_xml/

9. Holtkamp, Annette and Mele, Salvatore and Simko, Tibor and Smith, Tim, Realizing the dream of a global digital library in High-Energy Physics, CERN-OPEN-2010-019 